\title{
Strates
}

STRATES Matériaux pour la recherche en sciences sociales

9 | 1997

Crises et mutations des territoires

\section{Quelques éléments sur les difficultés d'insertion professionnelle des jeunes sans qualification dans l'agglomération toulousaine}

\section{Corinne Siino}

\author{
(2) OpenEdition \\ Journals \\ Édition électronique \\ URL : http://journals.openedition.org/strates/615 \\ DOI : $10.4000 /$ strates. 615 \\ ISSN : $1777-5442$ \\ Éditeur \\ Laboratoire Ladyss
}

Édition imprimée

Date de publication : 30 septembre 1997

ISSN : 0768-8067

Référence électronique

Corinne Siino, «Quelques éléments sur les difficultés d'insertion professionnelle des jeunes sans qualification dans l'agglomération toulousaine », Strates [En ligne], 9 | 1997, mis en ligne le 19 octobre 2005, consulté le 10 décembre 2020. URL : http://journals.openedition.org/strates/615 ; DOI : https:// doi.org/10.4000/strates.615

Ce document a été généré automatiquement le 10 décembre 2020.

Tous droits réservés 


\title{
Quelques éléments sur les difficultés d'insertion professionnelle des jeunes sans qualification dans l'agglomération toulousaine
}

\author{
Corinne Siino
}

1 Les analyses du chômage des jeunes montrent que leurs difficultés d'insertion sur le marché du travail ne correspondent pas seulement à une inadaptation du système de formation aux éventuels débouchés dans l'emploi. La dernière décennie a vu se multiplier les efforts des pouvoirs publics (Etat et Collectivités locales) et du secteur privé pour harmoniser et rationaliser le système de la formation : regroupements de filières dans des établissements, effort d'harmonisation du contenu et du niveau des diplômes... Les évaluations concernant le niveau d'adéquation entre les formations proposées et les emplois existants montrent que les acteurs intervenant dans le champ de la formation proposent des prestations de mieux en mieux adaptées aux niveaux de compétence requis par les emplois vers lesquels elles sont supposées déboucher. Cependant, malgré tous les efforts de modernisation du système de formation, et malgré les aides accordées par l'Etat aux entreprises qui acceptent de former ou de reconvertir des demandeurs d'emploi ${ }^{1}$, les jeunes les moins diplômés sont toujours les plus touchés par le chômage. La multiplication des statuts, la diversification du système de rémunération - en particulier dans le cadre de la formation en alternance ${ }^{2}-$ représentent au mieux des opportunités d'insertion temporaire, mais rarement une porte vers l'emploi durable. A l'issue d'un contrat à durée déterminée les jeunes se retrouvent souvent en situation de chômage de durée variable suivant leur niveau de qualification, leur âge, leur possibilité de mobilité, leur situation familiale, mais aussi suivant les potentialités du contexte socio-économique local où ils effectuent leur recherche d'emploi.

2 La dimension locale des marchés du travail n'est pas à négliger dans une réflexion sur les problèmes sectoriels et territoriaux du chômage des jeunes peu qualifiés. L'intérêt 
porté à cette dimension permet notamment d'aborder la question de l'efficacité des dispositifs publics de lutte contre le chômage en rapport avec un marché du travail déterminé. Le fonctionnement $\mathrm{du}$ dispositif de Préparation à la qualification et à l'emploi (PAQE) en région toulousaine en donne l'exemple.

Evolution du marché du travail toulousain (1975-1992)

3 Depuis la fin des années 1960 jusqu'à la fin des années 1980, le marché du travail toulousain a été de plus en plus pourvoyeur d'emplois tertiaires, avec une part très importante d'offres très qualifiées ${ }^{3}$. Ces changements ont été accompagnés de restructurations socio-professionnelles importantes (plus forte représentation des catégories moyennes et supérieures, recul des catégories les moins qualifiées chez les ouvriers et les employés). A partir de la décennie 1990, «les difficultés économiques... se sont amplifiées, touchant des secteurs d'activité auparavant épargnés et aggravant la situation de ceux qui étaient déjà atteints par la récession ${ }^{4}$."

4 Selon l'INSEE, à partir de 1992, l'emploi privé, qui enregistrait une forte progression de ses effectifs entre 1985 et $1990^{5}$ (+ 28357 emplois salariés dans la zone d'emploi de Toulouse), subit un brusque retournement. Les secteurs qui accueillaient traditionnellement les jeunes peu qualifiés, les apprentis, les immigrés, ne jouent plus leur rôle intégrateur dans l'emploi. Dans le bâtiment, la fin des grands chantiers (travaux du métro) et les restrictions budgétaires des collectivités territoriales s'ajoutent à l'arrêt des constructions de bâtiments neufs autres que les logements. Les entreprises du bâtiment ont supprimé plus de 2300 emplois dans le département depuis 1992 et ont répercuté leurs difficultés sur le secteur de la production de matériaux (tuiles et briques). Le commerce alimentaire de gros ou de détail, qui s'est maintenu jusqu'en 1992, connaît d'importantes restructurations par disparition ou rachat des unités de distribution. La consommation non alimentaire baisse avec la diminution de la consommation des ménages. Les effectifs d'emplois qui augmentaient jusqu'en 1991 stagnent depuis cette date, situation qui masque un important phénomène de créations-disparitions d'entreprises.

5 Les entreprises de haute technologie, considérées comme le moteur du dynamisme et de la croissance de l'agglomération, connaissent elles aussi des difficultés, qui ont des répercussions sur la sous-traitance (mécanique) et les prestataires de services (informatique, électronique). Enfin, dans les secteurs de la biotechnologie, de l'électronique et de l'informatique, la crise s'est manifestée sous forme de licenciements dans des sociétés importantes (Euro-Hermespace, Giat-Industrie et Thompson), par un chômage partiel, des aménagements d'horaires, et surtout par une externalisation importante de services auparavant intégrés dans les entreprises (nettoyage, messagerie), ou par la sous-traitance de certaines parties de contrat à d'exemployés qui voient ainsi leurs conditions de travail et leur statut professionnel modifiés. Pour les petites sociétés de services et de sous-traitance, les conséquences de la crise sont le rachat en totalité ou en partie par des sociétés plus importantes ${ }^{6}$.

6 Les récentes évolutions du marché du travail local qui viennent d'être décrites s'accompagnent d'une hausse du chômage (tableau 1). L'augmentation des demandeurs d'emploi en fin de mois (DEFM, ANPE) dans l'agglomération toulousaine est continue entre 1985 et 1989. Elle s'infléchit entre 1989 et 1990 pour connaître ensuite une accélération brutale. 1990 marque une rupture avec la situation antérieure puisque le nombre de DEFM augmente de 12000 en trois ans. De plus, celui des demandeurs inscrits depuis plus d'un an enregistre, entre 1992 et 1993, une augmentation 
équivalente à celle de 1990 à 1992 . Le poids de cette catégorie par rapport à l'ensemble des DEFM passe ainsi de 30,8\% en 1990 à 35,85\% en 1993. Le problème du chômage s'est donc nettement aggravé depuis 1990 et le chômage de longue durée pénalise surtout les actifs peu qualifiés.

7 Dans ce contexte, la situation des jeunes de moins de 25 ans est un peu particulière. Ils sont moins nombreux à être inscrits au chômage en 1993 qu'en 1985 ; mais si la baisse fut continue de 1985 à 1990, les fluctuations récentes - augmentation entre 1990 et 1992 puis légère diminution de 1992 à 1993 - les désignent comme particulièrement sensibles aux évolutions du marché du travail local. Parmi les facteurs qui sont à l'origine de ces évolutions contrastées, il en est un qui pose question actuellement: quels sont les effets des mesures d'aide à l'emploi et à l'insertion professionnelle des jeunes peu qualifiés? L'étude réalisée sur l'impact du dispositif PAQE donne quelques indications à ce sujet.

Tableau 1 - Les demandeurs d'emploi en fin de mois dans l'agglomération toulousaine

\begin{tabular}{|c|c|c|c|c|c|}
\hline & \multicolumn{2}{|c|}{ DEFM moins de 25 ans } & \multirow[t]{2}{*}{ Total DEFM } & \multirow{2}{*}{$\begin{array}{c}\text { dont DEFM } \\
\text { de plus d'un an }\end{array}$} & \multirow{2}{*}{$\begin{array}{l}\text { \% moins } 25 \text { ans } \\
\text { dans les DEFM }\end{array}$} \\
\hline & Total & dont femmes & & & \\
\hline 1985 & 9743 & 5285 & 26554 & 7664 & 36,7 \\
\hline 1986 & 9553 & 5307 & 28833 & 8044 & 33,1 \\
\hline 1987 & 9535 & 5379 & 30989 & 8822 & 30,8 \\
\hline 1988 & 9284 & 5276 & 32393 & 9297 & 28,7 \\
\hline 1989 & 8585 & 4941 & 33297 & 10394 & 25,8 \\
\hline 1990 & 7795 & 4471 & 32803 & 10103 & 23,8 \\
\hline 1991 & 8750 & 4881 & 38892 & 12417 & 22,5 \\
\hline 1992 & 9153 & 4937 & 42994 & 13897 & 21,3 \\
\hline 1993 & 8944 & 4685 & 44786 & 16057 & 20,0 \\
\hline
\end{tabular}

Source ANPE - DRTE

L'aide à l'insertion des jeunes les moins qualifiés par le dispositif PAQE

8 Ce dispositif a été une expérience de contractualisation entre l'Etat et les acteurs locaux intervenant dans le champ de la formation professionnelle à destination de jeunes peu qualifiés (niveaux V et VI) ${ }^{7}$. Décidé le 8 janvier 1992 par le gouvernement, il a pris fin en juillet 1994 ; il avait pour but de rendre effectif l'accès à une formation qualifiante ou à un emploi. Les stages proposés avaient un triple objectif : évaluer, pour chaque jeune, ses acquis minima, sa situation sociale (contexte familial, santé...), sa motivation pour un projet de formation en alternance ou pour une recherche d'emploi ; lui donner la 
maîtrise des savoirs de base : lire, écrire, compter, raisonner, communiquer, traiter des informations, travailler en équipe, prendre des initiatives ; lui faire découvrir plusieurs métiers, secteurs ou situations professionnelles pour qu'il puisse s'orienter vers la préparation d'une qualification ou la recherche ciblée d'un emploi.

L'analyse des parcours d'insertion effectués dans le cadre du dispositif est fondée sur les statistiques disponibles à l'échelle de la zone d'emploi pour l'année 1992 et sur la synthèse des enquêtes réalisées auprès de responsables d'organismes prestataires de formations, de chargés de mission assurant la relation entre les stagiaires et les entreprises, et d'acteurs sociaux en relation avec les jeunes chômeurs.

Les caractéristiques du public entré dans ce dispositif dans la région toulousaine sont semblables à celles qu'on observe ailleurs : prédominance masculine, moyenne d'âge relativement élevée (près de la moitié des jeunes ont entre 22 et 26 ans), niveaux scolaires très faibles, expérience professionnelle à peu près inexistante, difficultés sociales ou de santé qui excluent l'idée d'une embauche immédiate. Dans l'agglomération toulousaine comme dans d'autres villes françaises, les problèmes d'insertion, plus importants pour les jeunes qui habitent des quartiers à forte concentration de chômeurs et de population maghrébine, relèvent pour partie de critères objectifs tels que l'âge, le niveau réel de qualification, et pour partie d'une relation complexe entre la qualification de "territoires en difficulté » et les caractéristiques supposées des populations qui y vivent.

Des handicaps propres aux individus, ou inhérents à leur situation familiale et sociale, mettent en évidence l'existence de deux niveaux auxquels doivent jouer les actions visant à ramener ces publics vers le champ de l'emploi : l'acquisition d'une formation et l'aide à la resocialisation, sans que les deux soient nécessairement dissociées, car l'obstacle majeur est souvent constitué par le cumul de "freins à l'insertion». Cependant, si les programmes de réinsertion sociale tiennent compte de la diversité des handicaps, l'aide à l'insertion dans l'emploi assimilée en partie à un moyen pour atténuer des problèmes sociaux comporte deux dangers: faire apparaître comme réfractaires à tout effort d'insertion ceux qui n'auraient pas été insérés sur le marché du travail ; ne pas introduire de distinction, dans la perception et la prise en charge des handicaps sociaux, entre des jeunes encore bien «loin " d'une situation d'embauche pour lesquels l'urgence est une prise en charge sociale et médicale, et des jeunes dont les problèmes de socialisation ou de formation peuvent être en partie surmontés grâce à l'aide apportée par ce dispositif.

12 L'analyse des résultats obtenus à mi-parcours pour les jeunes en formation révèle un bilan mitigé sur le plan quantitatif (tableaux 2, 3 et 4).

Tableau 2 - Distribution des sorties du dispositif PAQE : bilan général à mi-parcours

\begin{tabular}{|c|c|c|}
\hline Résultats à mi-parcours (31/12/1993) & Nombre & $\%$ \\
\hline Abandon & 532 & 54,0 \\
\hline Formation & 129 & 13,1 \\
\hline Emploi & 167 & 16,9 \\
\hline
\end{tabular}




\begin{tabular}{|l|l|l|}
\hline En attente de formation ou d'emploi & 155 & 16,0 \\
\hline \hline Total des personnes entrées dans le dispositif & 983 & 100 \\
\hline
\end{tabular}

Source DRFP Toulouse, 1994

Tableau 3 - Les sorties en formation

\begin{tabular}{|l|l|l|}
\hline Résultats à mi-parcours (31/12/1993) & Nombre & $\%$ \\
\hline \hline Contrat d'apprentissage & 33 & 25,6 \\
\hline \hline Contrat de qualification & 20 & 15,5 \\
\hline \hline Formation CFI (pré-qualification ou qualification) & 55 & 42,6 \\
\hline \hline Autres actions d'insertion ou de formation & 21 & 16,3 \\
\hline \hline Total & 129 & 100 \\
\hline
\end{tabular}

Source DRFP Toulouse, 1994

Tableau 4 - Les sorties en emploi

\begin{tabular}{|l||l|l|}
\hline Résultats à mi-parcours (31/12/1993) & Nombre & $\%$ \\
\hline \hline Contrat à durée indéterminée & 51 & 30,5 \\
\hline \hline Contrat à durée déterminée de plus de 7 mois & 13 & 7,8 \\
\hline \hline Contrat à durée déterminée de moins de 6 mois & 30 & 18,0 \\
\hline \hline CES & 73 & 43,7 \\
\hline \hline Total & 167 & 100 \\
\hline
\end{tabular}

Source DRFP Toulouse, 1994

Plus de la moitié ont abandonné : les départs nombreux en phase initiale (0 à 300 heures dans le dispositif) ${ }^{8}$ résultent en grande partie d'une agrégation apparemment trop arbitraire des jeunes possédant déjà un savoir et des expériences professionnelles avec des jeunes ayant un niveau minimum de scolarisation; $30 \%$ ont pu obtenir un emploi ou une autre formation : pour ces jeunes-là, le dispositif PAQE a joué son rôle dans une logique de " parcours "; les autres sont de nouveau dans les files d'attente sur le marché du travail. 

locaux d'entreprise, le commerce de détail et le gardiennage, bien que caractérisées par un contingent important d'emplois de faible niveau de qualification, ne sont pas pour autant intéressées par des publics jeunes et peu qualifiés. Elles recherchent avant tout des actifs pouvant s'accommoder d'une importante flexibilité horaire et disposant de moyens de locomotion personnels. La plupart des postes proposés correspondent à des emplois où il n'y a pas de progression dans la qualification. La difficulté d'établir des projets professionnalisés et le manque de perspective par rapport à un métier sont donc peu attractifs et peu motivants pour certains jeunes actifs qui souhaitent acquérir une qualification. Pour d'autres, l'apprentissage de la socialisation, l'acquisition d'un niveau de connaissances générales minimum, et le travail en entreprise, exigent une période d'adaptation difficilement compatible avec la conception de la rémunération et de la productivité dans ces branches du secteur privé.

De manière générale, les petites entreprises - de 1 à 10 salariés - ont été les plus réceptives à l'accueil de jeunes en difficulté. Nombreuses dans le tissu économique, leur sensibilisation aux problèmes d'emploi de la population tient à leur vulnérabilité propre et à l'origine souvent locale de leur créateur. Malgré leur réticence à cause des coûts et des lourdeurs administratives, elles participent à la formation en alternance et quelquefois embauchent des jeunes très peu qualifiés. 
19 A travers les difficultés des jeunes peu qualifiés à s'insérer sur le marché du travail toulousain, les effets des dispositifs d'aide à l'insertion de ce type d'actifs semblent en partie dépendre des structures du marché local du travail. Dans l'agglomération toulousaine, les entreprises des secteurs de haute technologie ont aujourd'hui externalisé et sous-traité la plus grande part des emplois peu qualifiés autrefois intégrés dans leurs établissements. Compte tenu des restrictions des marchés et de la très forte concurrence dans des secteurs comme le nettoyage, l'entretien des espaces verts ou la maintenance, ces sous- traitants emploient des actifs immédiatement productifs qui permettent une rentabilité maximale de l'entreprise.

Dans ces conditions, le problème de l'insertion des actifs en difficulté sur le marché du travail est reporté sur le secteur associatif, les entreprises ou associations d'insertion, ce qui renforce les cloisonnements et les inégalités entre les différents segments du marché du travail local.

\section{NOTES}

1.Exonération des cotisations sociales « employeur » qui vont de $25 \%$ à $100 \%$ des charges patronales.

2. Stages et contrats de formation en entreprise et emplois aidés dans le secteur non marchand.

3. Ces évolutions ont été déjà largement mises en évidence dans les publications de l'INSEE, mais aussi dans les travaux de chercheurs qui se sont intéressés à l'évolution socio-économique et professionnelle de l'agglomération.

4. « La conjoncture en Midi-Pyrénées », Premier bilan économique de 1992, INSEE, mars 1993.

5. Essentiellement due aux secteurs suivants : industrie des biens d'équipement, transports-télécommunications services marchands (cf. L'emploi en Midi-Pyrénées du 1er janvier 1986 au premier janvier 1990, Les dossiers Midi-Pyrénées, 46, INSEE, 1991).

6. Rachat de Vérilog (informatique), de Bio Europe (recherche et production pharmaceutique), de CEIS (électronique appliquée au spatial), d'Opsia (implants et produits de chirurgie oculaire)... (cf. La Dépêche du Midi, 25/01/1993.

7. Niveau V : CAP, BEP, ou abandon d'un cycle long avant la terminale. Niveau V bis : sortie de troisième ou d'un second cycle court avant l'année de terminale. Niveau VI : sortie du premier cycle du second degré.

8. $66,7 \%$ de ces sorties correspondent à un abandon, quelques-unes à l'entrée dans une formation ou à un emploi hors du dispositif. 


\section{RÉSUMÉS}

Une analyse de l'insertion des jeunes peu qualifiés sur le marché du travail toulousain à travers le dispositif PAQE, montre que, malgré l'existence d'un potentiel d'emplois non négligeable, il leur est difficile de trouver une place.

Some insights into vocational insertion difficulties of unskilled young people in the Toulouse agglomeration

The analysis of the program PAQE results in Toulouse metropolitan area is showing young low skilled difficulties to find a job even if employments exist.

\section{INDEX}

Mots-clés : jeunes, faible qualification, insertion, marché du travail, Toulouse

Keywords : youth, low skill, labour market

\section{AUTEUR}

\section{CORINNE SIINO}

Corinne SIINO est maître de conférences à l'Université de Toulouse le Mirail. Ses recherches, menées au sein du Centre Interdisciplinaire d'Études Urbaines, portent sur les rapports entre recompositions sociales et économiques des marchés du travail et développement urbain. 